\title{
Graviton mass from close white dwarf binaries detectable with LISA
}

\author{
Asantha Cooray* and Naoki Seto ${ }^{\dagger}$ \\ California Institute of Technology, Mail Code 130-33, Pasadena, California 91125, USA
}

(Received 2 November 2003; published 7 May 2004)

\begin{abstract}
The arrival times of gravitational waves and optical light from orbiting binaries provide a mechanism to understand the propagation speed of gravity when compared to that of light or electromagnetic radiation. This is achieved with a measurement of any offset between the optically derived orbital phase and that derived from gravitational wave data, at a specified location of one binary component with respect to the other. Using a sample of close white dwarf binaries (CWDBs) detectable with the Laser Interferometer Space Antenna and optical light curve data related to binary eclipses from meter-class telescopes for the same sample, we determine the accuracy to which orbital phase differences can be extracted. We consider an application of these measurements involving a variation of the speed of gravity, when compared to the speed of light, due to a massive graviton. For a subsample of $\sim 400 \mathrm{CWDBs}$ with high signal-to-noise ratio gravitational wave and optical data with magnitudes brighter than 25 , the combined upper limit on the graviton mass is at the level of $\sim 6 \times 10^{-24} \mathrm{eV}$. This limit is two orders of magnitude better than the present limit derived by Yukawacorrection arguments related to the Newtonian potential and applied to the Solar System.
\end{abstract}

DOI: 10.1103/PhysRevD.69.103502

PACS number(s): 98.80.Es, 95.85.Nv, 98.35.Ce, 98.70.Vc

\section{INTRODUCTION}

In a recent paper, we discussed the optical follow-up study of close white dwarf binaries (CWDBs) that will be detectable with the Laser Interferometer Space Antenna (LISA) mission [1]. Recent estimates suggest that close to 3000 binaries will be detected via gravitational waves (GWs) at frequencies above $3 \mathrm{mHz}$, with most of them restricted to the frequency range between $3 \mathrm{mHz}$ and $6 \mathrm{mHz}$ [2-4]. These binaries will be localized only to the accuracy of $\sim$ one square degree with a three-year LISA observation based on the Doppler effect related to the orbit around the Sun and the amplitude modulation due to the rotation of LISA detectors. For precise location of an individual binary, optical observations are required; an easy identification of the binary is facilitated by the presence of eclipses in the optical light curve with a period twice the GW period. Considering the impact on astronomy and astrophysics, in general, one can certainly expect detailed optical follow-up observations of LISA binaries [1]. In addition to CWDB related physics, such as tidal heating, using both the optical light curve and gravitational wave data, we can also extract important information related to certain fundamental parameters in physics. We discuss such a possibility in the present paper by considering observational prospects in the LISA era.

In addition to a localization of the direction from which GWs are emitted, with gravitational waves, LISA also allows a measurement of the binary distance, the chirp mass, the binary orbital inclination angle, and the binary orbital phase (modulus $\pi$ ). The optical follow-up observations also allow a measurement of the orbital phase. In general, one expects the orbital phase measured with gravity wave data to agree precisely with that of the optical light curve. A difference in the two is expected only if opticals light travels at a different

\footnotetext{
*Email address: asante@tapir.caltech.edu

${ }^{\dagger}$ Email address: seto@tapir.caltech.edu
}

speed from that of gravity, which determines the propagation of gravitational waves. While a difference is not expected, a potential non-zero mass related to the graviton particle can lead to a slight difference. Thus, a comparison of the orbital phase related to an optical eclipsing light curve of CWDBs with the phase determined from LISA data will allow a constraint on the graviton mass.

Note that previous studies have considered such a possibility, again with respect to the Laser Interferometer Gravitational Wave Observatory (LIGO) and LISA data [5-8]. While one of the considered methods involves the dispersion of the gravity waves alone, as a function of the frequency $[5,6]$, the comparison between optical or electromagnetic data and gravity waves is expected to provide an improved constraint. Previous analyses, however, considered only a handful of objects which are already known to be eclipsing binaries in optical data and are expected to be GW sources detectable with LISA (e.g., 4U1820-30) [8]. The present study considers a sample of CWDBs detectable and locatable with LISA above its confusion noise and bright enough optically for follow-up studies with a few meter-class telescopes.

The paper is organized as follows. In Sec. II, we briefly discuss observations with LISA, requirements for an optical follow-up study and, then, consider the extent to which orbital phases of individual binaries can be determined. In Sec. III, we put these measurements in the context of an improved limit on the graviton mass. We conclude with a summary in Sec. IV.

\section{CLOSE WHITE DWARF BINARIES}

First, we will review CWDB detection with LISA in gravitational waves and then move on to discuss aspects related to the optical light curve. Our focus here is related to the phase measurement related to the binary orbit, while issues related to the CWDB localization with LISA data and optical follow-up observations are discussed in Ref. [1], fol- 
lowing initial calculations in Refs. $[9,13,14]$.

\section{A. LISA observations}

Briefly, with gravitational waves, one observes two components given by the quadrupole approximation in the principal polarization coordinate [10]

$$
\begin{aligned}
& h_{+}(t)=A \cos \left[2 \pi\left(f+\frac{1}{2} \dot{f} t\right) t+\varphi(t)\right] \times\left[1+(\hat{L} \cdot \hat{n})^{2}\right], \\
& h_{\times}(t)=-2 A \sin \left[2 \pi\left(f+\frac{1}{2} \dot{f} t\right) t+\varphi(t)\right] \times(\hat{L} \cdot \hat{n}),
\end{aligned}
$$

where $\hat{n}$, given by $\left(\theta_{S}, \phi_{S}\right)$, and $\hat{L}$ are unit directional vectors to the binary, from the observer, and the angular momentum of the binary, respectively. Here, $\varphi(t)$ is the phase resulting from the Doppler phase due to the revolution of LISA around the Sun:

$$
\varphi(t)=2 \pi f R \sin \theta_{S} \cos \left[\frac{2 \pi t}{1 \mathrm{yr}}-\phi_{S}\right]+\varphi_{0}
$$

where $R=1 \mathrm{AU}$ and $\varphi_{0}$ is an integral constant. The principal polarization coordinate is determined by two orthonormal vectors $\hat{X}$ and $\hat{Y}$ that are normal to the direction of the source $\hat{n}$ through

$$
\begin{aligned}
& \hat{X}=\frac{\hat{n} \times \hat{L}}{|\hat{n} \times \hat{L}|}, \\
& \hat{Y}=\hat{n} \times \hat{X} .
\end{aligned}
$$

CWDBs are expected to have circular orbits due to the tidal interaction in their early evolutional stage. The amplitude of the wave is given by

$$
A=\frac{5}{96 \pi^{2}} \frac{\dot{f}}{f^{3} D}
$$

where $D$ is the distance to the GW source. Note that the GW frequency ( $=2 / P_{\text {orb }}$ where $P_{\text {orb }}$ is the orbital period) for a circular orbit is related to the total mass $M_{1}+M_{2}$ and the separation $a$ of the binary via

$$
\begin{aligned}
f & =2 \sqrt{\frac{G\left(M_{1}+M_{2}\right)}{4 \pi^{2} a^{3}}} \\
& \approx 3.5 \times 10^{-3}\left(\frac{M_{\text {tot }}}{0.9 M_{\odot}}\right)^{1 / 2}\left(\frac{a}{10^{5} \mathrm{~km}}\right)^{-3 / 2} \mathrm{~Hz},
\end{aligned}
$$

while the time variation of this frequency is

$$
\begin{aligned}
\dot{f}= & \frac{96 \pi^{8 / 3}}{5} \tilde{M}^{5 / 3} f^{11 / 3} \approx 1.210^{-16}\left(\frac{\tilde{M}}{0.4 M_{\odot}}\right)^{5 / 3} \\
& \times\left(\frac{M_{\mathrm{tot}}}{0.9 M_{\odot}}\right)^{11 / 6}\left(\frac{a}{10^{5} \mathrm{~km}}\right)^{-11 / 2}
\end{aligned}
$$

when the chirp mass is given by $\tilde{M}=M_{1}^{3 / 5} M_{2}^{3 / 5} /\left(M_{1}\right.$ $\left.+M_{2}\right)^{1 / 5}$ and the total mass $M_{\text {tot }}=M_{1}+M_{2}$.

With gravitational waves, one can estimate a total of 8 independent quantities: $A, f, \dot{f}, \varphi_{0}$, location $(\vec{n}, 2$ parameters), and the direction of the angular momentum, $\vec{L}$ (2 parameters). At higher $\mathrm{GW}$ frequencies, one can also extract meaningful information on the second derivative of the frequency $\ddot{f}$. The orbital inclination angle is given by $\cos ^{-1}(\vec{n}$ $\cdot \vec{L}$ ). In terms of physical quantities of interest, with $A, f, \dot{f}$, one extracts $D$ and $\tilde{M}$; in addition to these parameters, one can also constrain a combination of $M_{\text {tot }}$ and $a$ with Eq. (5). Note that relations (4) and (6) are given for Newtonian point particle systems as the correction related to the finite size of the binary is not significant.

With parameters related to $\hat{n}$ and $\hat{L}$, one can reconstruct the orbital phase, say at a certain time $t$, which we define as

$$
\phi(t) \equiv \tan ^{-1}\left[\frac{\hat{r}(t) \cdot \hat{X}}{\hat{r}(t) \cdot \hat{Z}}\right],
$$

where $\hat{Z}=\hat{L} \times \hat{X}$ and $\hat{r}(t)$ is the unit vector between the two masses. This vector changes as a function of time as the binary components orbit with respect to a common center of mass.

For the present discussion, we extend the calculation in Ref. [1] to study how well the orbital phase in each of the LISA detectable CWDBs can be determined. In order to generate the necessary representation of LISA observations, we make use of the sample studied in [1] based on the population synthesis code used by Ref. [11]. This binary sample is distributed in the galaxy following

$$
\rho(R, z)=\rho_{0} \operatorname{sech}\left(\frac{|z|}{z_{0}}\right)^{2} \exp \left(-\frac{R}{R_{0}}\right),
$$

where $z_{0}=200 \mathrm{pc}$ and $R_{0}=2.5 \mathrm{kpc}$ [12]. For the sample of $\sim 3000$ binaries, we now randomly assign an orientation $\vec{L}$. This means that the inclination $\cos i$ is distributed uniformly in $0 \leqslant \cos i \leqslant 1$. For an optical eclipse to be observable, we require that the cosine of the inclination angle be less than a minimum such that $\cos i \leqslant \cos i_{\min } \equiv\left(R_{1}+R_{2}\right) / a$. For simplicity, here, we assume that the binaries have equal mass. For a given orbital frequency and a chirp mass, we calculate the ratio $\left(R_{1}+R_{2}\right) / a$ as a function of $q \equiv M_{1} / M_{\text {tot }}$ using a typical mass-radius relation of white dwarfs given in Ref. [15]. We found that the ratio $\left(R_{1}+R_{2}\right) / a$ takes a minimum value at $q=0.5$ and increases only $\sim 10 \%$ at $q=0.3$ or $q=0.7$. This fractional change depends very weakly on the chirp mass. Considering the fact that the mass ratio $M_{1} / M_{2}$ for a 


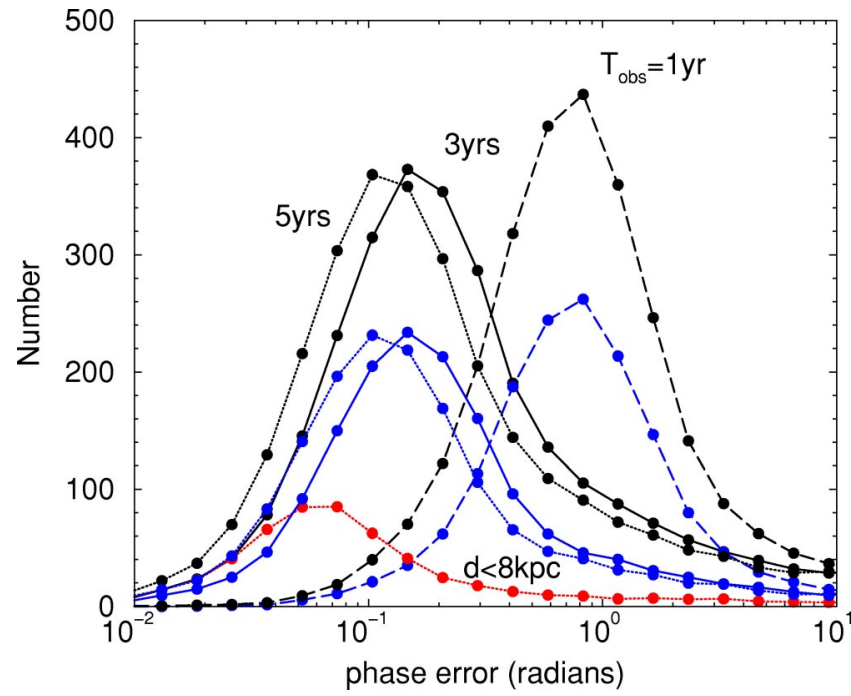

FIG. 1. The phase error distribution for GWs detectable with LISA for a sample $\sim 3000$ CWDBs, and assuming a total observational duration of 1,3 and $5 \mathrm{yr}$. The error in orbital phase is calculated following the Fisher matrix approach, with a total of eight parameters to be determined from gravity-wave data, including binary location. The lower curves are the errors in orbital phase for the CWDBs that are expected to show eclipses. For comparison, we also show phase errors related to the subsample, about $\sim 500$ objects, that are at distances below $8 \mathrm{kpc}$ and are expected to show eclipses in the optical light curve, under the condition that $\cos i$ $\leqslant\left(R_{1}+R_{2}\right) / a$, assuming a total observational duration of $5 \mathrm{yr}$. On average, the phase measurement with the nearby sample is better since the expected signal-to-noise ratio for gravitational wave detection is higher.

CWDB is expected to be close to 1 [16], our equal-mass assumption is conservative and safe.

Under these parameters, we extracted a subsample of objects from the total original sample that is expected to emit detectable GWs with LISA and also show eclipses in the optical light curve. Since $a$ typically is a few times $\left(R_{1}\right.$ $+R_{2}$ ), the probability of observing an eclipse is rather high and this subsample amounts to about 1400 objects with an overall probability of $\sim 0.45$. The subsample size is likely to be higher by up to $\sim 100$ objects when our assumption related to equal mass binaries is dropped.

The distribution of phase errors for this sample of $\sim 3000$ CWDBs detectable with LISA is shown in Fig. 1. The phase error is calculated using the Fisher matrix approach discussed in Ref. [13], where all 8 parameters related to these observations are derived simultaneously from GW data. As in Ref. [13], we use expressions related to LISA noise appropriate for the long wavelength limit, when compared to arm length, with a transfer function for the finite size of the arm length that subsequently corrects for the first assumption. This prescription is very effective for nearly monochromatic sources [17]. The noise curve in Ref. [18] is used here. We consider three cases with a total observational duration with LISA of 1, 3, and $5 \mathrm{yr}$. The lower set of lines show the phase error related to the subsample that is expected to show eclipses in the optical light curve, simply selected based on the inclination angle. This subsample can be optically iden- tified and localized precisely; however, assuming that the location is exactly known is not important when the observational duration is more than $2 \mathrm{yr}$ (see, e.g., Ref. [13] for details on parameter improvement when the binary location is assumed to be known precisely a priori).

As discussed in Ref. [8], the phase error related to GWs is simply given by

$$
\delta \phi_{\mathrm{GW}}=\frac{\alpha}{2}\left(\frac{S}{N}\right)^{-1}\left[1+\mathcal{O}(S / N)^{-1}\right]
$$

where $\alpha$ is order unity or more and accounts for the fact that more than one parameter is to be determined from $\mathrm{GW}$ data. The factor of 2 in the above accounts for the fact that we are determining the phase error related to the binary orbit, which is $1 / 2$ the gravity-wave phase. The phase errors are generally at the level of $\sim 0.1$, when the observational duration is more than $3 \mathrm{yr}$. This is substantially smaller than what was previously suggested in the literature (cf. [7]). ${ }^{1}$

In Fig. 1, for reference, we also show the phase error related to the nearby CWDB sample (at distances below 8 kpc). There are roughly $\sim 1000 \mathrm{CWDBs}$ and we expect roughly $\sim 500$ of them to show eclipses that can be detected in the optical light curve. The phase error related to the nearby sample is better than for the whole sample and results from the fact that the signal-to-noise ratio for an individual detection is higher given the close distance.

Note that the orbital phase determination is substantially improved with observational durations, $T_{\text {obs }}$, at the level of 3 yr. While one generally expects the phase error determination to scale as $\sqrt{T_{\text {obs }}}$, there is a substantial improvement between one- and three-year durations beyond that implied by this simple scaling [13]. This increase results from the fact that significant degeneracies, between the binary location and other parameters, are broken with observational durations greater than $2 \mathrm{yr}$. We illustrate this in Fig. 2 where we show the phase error distributions for parameter determination that involve (i) all eight parameters, (ii) six parameters with location assumed to be known, and (iii) four parameters with both location and orbital orientation assumed to be known. The 1 yr curves with cases (i) and (ii) show the extent to which degeneracies affect the orbital phase determination. With location assumed to be known, for an observational duration of $1 \mathrm{yr}$, the degeneracy is broken and a substantial improvement in the orbital phase determination is achieved. In contrast, the curves for cases (i) and (ii) almost overlap when $T_{o b s}=3 \mathrm{yr}$ or $5 \mathrm{yr}$. These results are instructive in understanding how the impact of precise localization

\footnotetext{
${ }^{1}$ The phase error is independent of the timing error and the extent to which the period is known, as assumed in estimates by [7]. This can be simply understood by Fourier decomposing the light curve. The phase is simply related to the angle between a Fourier mode and an arbitrary vector, related to the location from which the phase is measured, and the extent to which this angle can be determined depends on the length of the Fourier mode, which in turn is determined by the signal-to-noise ratio of the light curve and not the timing accuracy.
} 


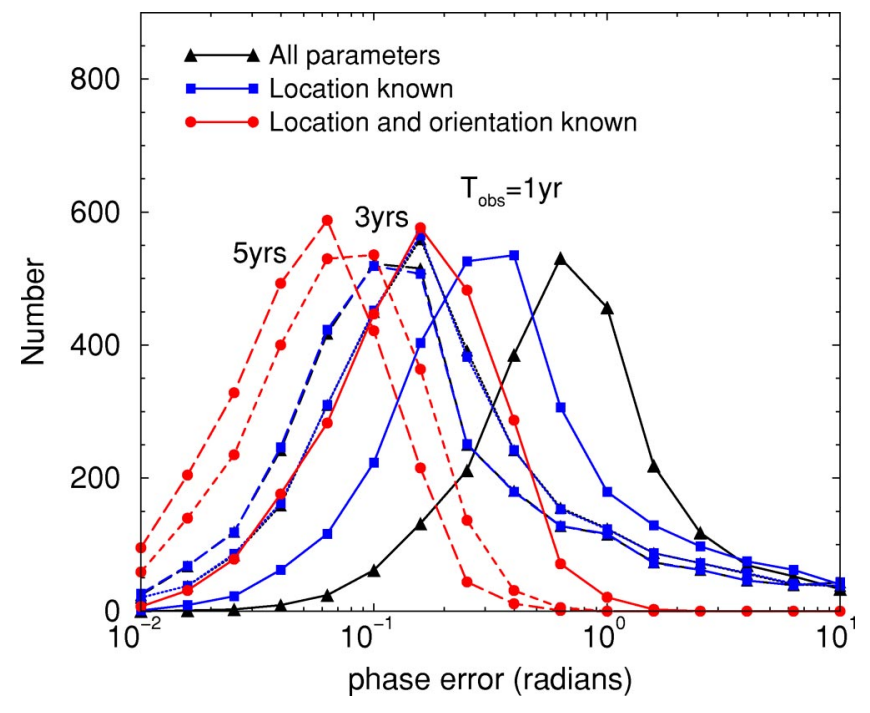

FIG. 2. We show the role of prior knowledge related to the binary location and orientation when the orbital phase errors are determined from GWs detectable with LISA for a sample $\sim 3000$ CWDBs, and, again, assuming a total observational duration of 1 (solid lines), 3 (dotted lines) and 5 (dashed lines) yr. The three sets of curves with triangles, squares and circles represent the cases where (i) no prior knowledge is available and all eight parameters are to be determined from the GW data, (ii) location is assumed to be known, and (iii) both location and orientation of the binary are assumed to be known, respectively. In general, the location and other parameters are highly correlated and precise localization leads to a substantial reduction in the accuracy of phase determination for observational durations of $2 \mathrm{yr}$ and less. For observational durations greater than $3 \mathrm{yr}$, the improvement in phase error related to a priori known location is not significant when compared to the case when all parameters, including location, are to be determined from the data. Curves for cases (i) and (ii) are almost overlapping for $T_{o b s}$ $=3 \mathrm{yr}$ and $5 \mathrm{yr}$. Additional degeneracies are broken when the binary orientation is also assumed to be known and this results in a better determination of the orbital phase. We do not pursue this possibility since it involves detailed modeling of the optical light curve.

(through optical identification) on parameter estimation depends on the observational duration of LISA.

Considering the observational situation relevant for this paper, we can implicitly assume that individual binary locations are determined precisely by the identification of optical counterparts. However, when we consider observational durations of order $3 \mathrm{yr}$ or more for GWs, the final graviton mass limit we calculate in the present paper is essentially independent of whether the binary location is assumed to be known or not in the GW phase measurement. One can obtain an improvement of a factor of 2 in the GW orbital phase when the orientation of the binary is also assumed to be known a priori. We do not pursue this possibility, however, since it involves detailed modeling of the optical light curve or obtaining additional data at different wavelengths of the electromagnetic radiation spectrum. For example, we might get limited information on the inclination angle by modeling the eclipse shape, but the polarization angle would not be determined.

\section{B. Optical data}

The optical magnitude distribution of the CWDB binary sample that is expected to be localized with LISA is discussed in Ref. [1]. Most optical magnitudes are at the level of 25 and fainter. Thus, we will restrict the discussion to only the subsample that is bright. Similarly, the extent to which the phase angle can be determined from the optical light curve depends on the single-to-noise ratio of the optical light curve, and we write

$$
\delta \phi_{\mathrm{opt}}=\beta\left(\frac{S}{N}\right)^{-1}\left[1+\mathcal{O}(S / N)^{-1}\right]
$$

where now $\beta$ is again a parameter of order unity or more as we are trying to determine additional parameters from the optical data as well. Under the assumption that the period is known a priori, from the GW data, and the assumption that the time derivative of the period is zero, which is a very safe assumption since $\dot{P} \sim 10^{-10} s s^{-1}$ for binaries at $3 \mathrm{mHz}$ with chirp mass $\sim 0.45 \mathrm{M}_{\odot}, \beta$ is generally at the level of 1 or slightly above. Since the observational duration of GWs, $T_{\mathrm{obs}, \mathrm{GW}} \sim 3 \mathrm{yr}$ or more, is larger than the duration of optical observations ( $\sim 100 \mathrm{~min})$, our assumption of neglecting $\dot{P}$ for optical analysis is safe, although we include $\dot{P}$, through $\dot{f}$, in estimating parameters related to GWs.

For a meter-class telescope, with an integration time of $t_{\text {int }}$, the expected signal-to-noise ratio can be written as

$$
\frac{S}{N}=\frac{S_{\mathrm{WD}} t_{\text {int }}}{\sqrt{S_{\mathrm{WD}} t_{\text {int }}+S_{\text {sky }} t_{\text {int }}+S_{\text {det }} t_{\text {int }}}},
$$

where $S_{\mathrm{WD}}, S_{\text {sky }}$, and $S_{\text {det }}$ are the number of source, sky background, and detector photons, respectively, per unit time. For a star of magnitude $m$, imaged with a telescope of diameter $D$ and efficiency $\epsilon$, the number of photons, in a second, is

$$
S_{\mathrm{WD}}=7.5 \times\left. 10^{2}\left(\frac{\epsilon}{0.5}\right)\left(\frac{10^{-2 / 5 m}}{10^{-10}}\right)\right|_{m=20}\left(\frac{D}{4 \mathrm{~m}}\right)^{2} .
$$

In the limit that sky and detector backgrounds are not important, the signal-to-noise ratio, for a 25 -s integration is

$$
\frac{S}{N}=\left.120\left(\frac{\epsilon}{0.5}\right)^{1 / 2}\left(\frac{10^{-1 / 5 m}}{10^{-5}}\right)\right|_{m=20}\left(\frac{D}{4 \mathrm{~m}}\right)\left(\frac{t_{\text {int }}}{25 \mathrm{~s}}\right)^{1 / 2} .
$$

In reality, at these faint magnitudes, the signal-to-noise ratio is, however, not determined only by the flux from the object, but rather by the sky and the detector background, and the true signal-to-noise ratio, for a $25 \mathrm{~s}$ observation, is at the level of 76 for $m=20,12$ for $m=22.5$, and $\sim 1.5$ for $m$ $=25$, in the case of the 4-m Cerro-Tololo and Kitt Peak telescopes of the National Optical Astronomical 


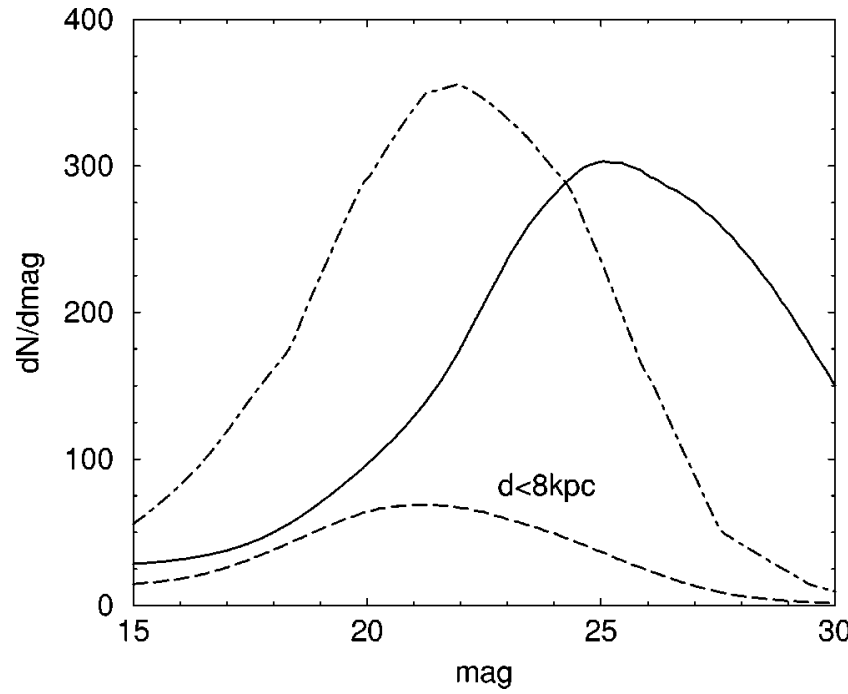

FIG. 3. The V-band magnitude distribution for the CWDB sample detectable with LISA and for which phase measurements may be possible. We have taken into account the spatial distribution in addition to extinction (solid line). The long-dashed line show the subsample at distances below $8 \mathrm{kpc}$. This subsample, on average, is brighter given the nearby distance and relatively low extinction, although the distribution is some what broad. The dash-dotted line shows the possible effects of the tidal heating when using the extreme model in [20].

Observatories. ${ }^{2}$ In our calculations, we use these numbers, but restrict our attention to the subsample at a distance below $8 \mathrm{kpc}$ and with magnitudes brighter than 25 ; with this additional restriction, the subsample reduces to about $\sim 400$ CWDBs, for which reliable phase measurements may be possible from both GWs, with LISA, and optical data.

Given the magnitude distribution of the CWDBs that are expected to show optical eclipses, we can estimate the expected distribution of errors in the orbital phase, determined from electromagnetic radiation. We summarize our results in Fig. 3 for the subsample of CWDBs, where we also include extinction [19], following the modeling in Ref. [1]. Most magnitudes are at the level of 25 and below, though for the sample with distances below $8 \mathrm{kpc}$, magnitudes are at the level of 22, albeit a broad distribution over the range of 20 to 25 . While magnitudes here are simply calculated based on white dwarf cooling, given the age distribution determined in Ref. [1], there is an additional effect that can potentially brighten CWDBs due to tidal heating by one white dwarf on the other due to their close separation [20]. The magnitude distribution gains a few magnitudes as shown in Fig. 3, but we do not consider this possibility given the complex physics associated with tidal heating such as the possibility that tidal heating is not uniform and will lead only to hot spots among the other CWDBs. However, we note that, from detailed follow-up observation of nearby CWDBs, one can obtain information that would potentially affect the orbital phase measurement from optical data, e.g., the brightness distribution of the surface of the white dwarf. Note also that inter-

\footnotetext{
${ }^{2}$ http://www.noao.edu/cgi-bin/scope/runiraf/ccdtime
}

acting binaries, such as AM CVn systems, would be more luminous, but they generally have smaller chirp masses (weaker gravitational wave signals), and estimation of their orbital phases from optical data would not be as simple as for CWDBs (see e.g., [7]).

\section{GRAVITON MASS}

With phase errors related to the GW and optical light curve data, one can consider a combined study involving the propagation speeds of gravity $\left(c_{g}\right)$ and light $(c)$. The first obvious application is to determined if $c_{g}=c$ and to study any departures from this. Compared to many other previous tests, this is an ideal scenario since one is directly comparing the gravitational radiation with optical light with a calibrated zero point with respect to a certain location of the orbit. The presence or the lack of any departure in the optically derived orbital phase from that of GWs can then be used to constrain some aspects related to the propagation that potentially make two speeds depart from each other.

As such a possibility, we consider the presence of a massive graviton such that the speed of gravity is modified:

$$
\begin{aligned}
c_{g} & =c\left[1-\frac{m_{g}^{2} c^{4}}{E^{2}}\right]^{1 / 2} \\
& \approx c\left[1-1 / 2 \frac{m_{g}^{2} c^{4}}{E^{2}}\right] .
\end{aligned}
$$

For the orbital binary, assuming a gravity wave frequency of $f\left(=2 / \mathrm{P}_{\text {orb }}\right)$ and at a distance $D$ the two phases are

$$
\begin{aligned}
\phi_{\mathrm{opt}} & =2 \frac{f D}{c}, \\
\phi_{\mathrm{GW}} & =\frac{f D}{c_{g}} .
\end{aligned}
$$

Simplifying with Eqs. (14) and defining $\Delta \equiv \phi_{\mathrm{GW}}-\phi_{\mathrm{opt}}$, one obtains the constraint that

$$
m_{g} c^{2}=h f\left[\frac{1}{2}\left(1+\frac{\pi f D}{c \Delta}\right)\right]^{-1 / 2} .
$$

In the absence of a measurable phase difference $\Delta=0$, an upper limit on $m_{g}$ can be obtained with the limit on $\Delta$ such that

$$
\Delta=\sqrt{\delta \phi_{\mathrm{GW}}^{2}+\delta \phi_{\mathrm{opt}}^{2}}
$$

In the limit that $\delta \phi_{\mathrm{GW}} \gg \delta \phi_{\mathrm{opt}}$, the limit on the graviton mass can be written in terms of the signal-to-noise ratio for detection of gravitational waves by LISA [8]:

$$
m_{g} c^{2}<\frac{h \sqrt{\alpha}}{\pi} \sqrt{\frac{\pi f c}{D}}\left(\frac{S}{N}\right)^{-1 / 2}
$$

and since the GW signal-to-noise ratio simply scales as $1 / D$, this limit is independent of the distance at which the binary is located [8]. In contrast the signal to noise ratio of electro- 
magnetic waves scales as $1 / D^{2}$, such that in the opposite limit, the limit on the graviton mass is no longer independent of the distance.

As shown in Fig. 1, and implied from Fig. 3, neither one of these assumptions, $\delta \phi_{\mathrm{GW}} \gg \delta \phi_{\mathrm{opt}}$ or $\delta \phi_{\mathrm{GW}} \ll \delta \phi_{\mathrm{opt}}$, however, is correct since the error to which both optical and gravity-wave phases are determined is at the same order. Thus, one should include both errors in estimating the limit on the graviton mass. For the present discussion, we make use of the sample at a distance below $8 \mathrm{kpc}$. The subsample is brighter and is within the magnitude limits comfortably reachable with few meter or more class telescopes that are currently available. We make use of a fraction of 400 objects with magnitudes brighter than 25 , and with distances below $8 \mathrm{kpc}$. Here, we neglect the uncertainty in the distance estimation related to LISA data since its error is in the second order. The individual distances are, however, statistically included by accounting for the distance distribution function based on our model assumption related to the spatial distribution following Eq. (8).

Note that the observations we consider are independent of the localization of the CWDBs since this can be achieved at a high signal-to-noise ratio by imaging at time intervals corresponding to expected minima and maxima in the optical light curve based on prior phase information from the gravity-wave data. To obtain the optical phase precisely, detailed sampling of the light curve is required and will involve imaging at time intervals of order $25 \mathrm{~s}$, given that the periods are mostly between 400 and $800 \mathrm{~s}$. The orbital phase errors related to optical and GW data are summarized in Fig. 4 for the subsample of CWDBs. The phase errors are generally at the level of $10^{-1}$ for GWs, while the orbital phase errors from the optical data follow a broad distribution. The error related to the combined phase difference is also shown in Fig. 4; it is calculated using physical properties of the subsample. In general, note that the phase error distributions related to GWs and optical data are not independent; the CWDBs with better determined orbital phases with GW data are expected to be nearby and, thus, to be bright optically. For these nearby ones, the orbital phases from optical light curve data are also better determined.

In the bottom plot of Fig. 4, we summarize the extent to which the graviton mass can be constrained from each of the CWDBs at distances below $8 \mathrm{kpc}$ and with magnitudes better than 25 . The one-sigma limit on the graviton mass is generally at the level of $10^{-22} \mathrm{eV}$. If phase information related to the whole sample can be combined for a single constraint, the combined limit by taking $\left(\sum_{i} 1 / \sigma_{i}^{2}\right)^{-1 / 2}$ improves to about $6 \times 10^{-24} \mathrm{eV}$ at the one-sigma level. This can be compared to current limits on the graviton mass. Based on the Yukawa correction to a Newtonian potential, in the presence of a massive graviton, the limit from Solar System dynamics is $4.4 \times 10^{-22} \mathrm{eV}$ [21], although the limit improves substantially to $2 \times 10^{-29} \mathrm{eV}$ with galaxy clusters at Mpc size scales $[22,23]$. The $90 \%$ confidence limit from the orbital decay of two-binary pulsars, again an indirect estimate of the graviton mass, is $7.6 \times 10^{-20} \mathrm{eV}$ [24]. The direct limits from GW and optical data are discussed in Refs. [7] and [8]; these limits are generally above the level we have discussed, as they
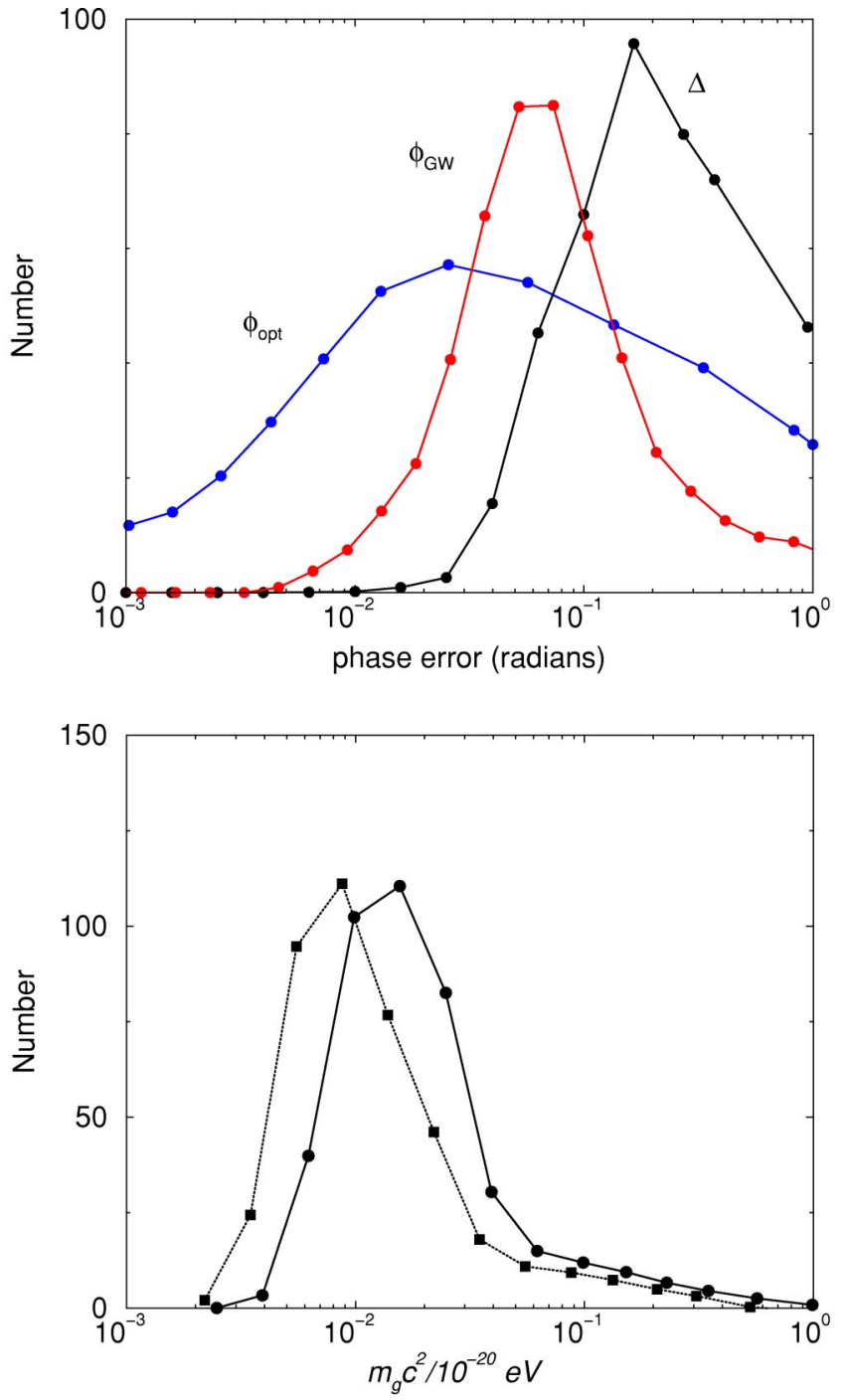

FIG. 4. Top: The error in orbital phase distribution for a subsample of $\sim 400 \mathrm{CWDBs}$ at distances below $8 \mathrm{kpc}$ and at magnitudes brighter than 25 . We show separately the orbital phase error from $\mathrm{GW}$ and optical data and the combined error related to the measurement of the phase difference in each of these cases. For each binary, we assume optical data of over 10 orbital cycles or roughly over a time interval of $\sim 6000 \mathrm{~s}$, and consider the signalto-noise measures as expected from a four-meter class telescopes [see Eqs. (11)-(13) and the discussion below Eq. (13)]. For the whole sample of $400 \mathrm{CWDBs}$, such a monitoring project takes a total of $\sim 90$ days assuming $8 \mathrm{~h}$ of useful observational time each day. Bottom: The distribution of graviton mass limits derived from the above sample, together with its distance and frequency distributions. The limit, for an individual CWDB, is generally at the level of $10^{-22} \mathrm{eV}$, but for the sample as a whole, this limit improves to about $\sim 6 \times 10^{-24} \mathrm{eV}$. The dotted line is the result considering only the phase errors of gravitational waves. The combined limit becomes $\sim 3 \times 10^{-24} \mathrm{eV}$.

usually involve either a single binary or different assumptions and estimates. Our limit, for the sample as a whole, is consistent with the best possible limit from LISA data implied in Ref. [8].

In general, we believe that the CWDB sample related to 
LISA provides a well controlled sample with adequate optical follow-up opportunities to obtain a reliable limit on the graviton mass as a whole. When obtained, such a limit will be independent of indirect assumptions and will be directly based on the propagation speeds of gravity and light. Finally we comment on a potential contaminant related to this measurement involving gravitational lensing of the background binaries by the foreground mass distribution. Note that the lensing probability in our galaxy has been measured to be at the level of $\tau \sim 10^{-6}$ [25], suggesting that the probability of observing a lensed CWDB is substantially small. Since gravitational waves from CWDBs have much lower frequencies $\left(\sim 10^{-2} \mathrm{~Hz}\right)$ than optical waves $\left(\sim 10^{16} \mathrm{~Hz}\right)$, the critical frequency for lensing with a mass $M_{\text {lens }}$ is $f_{\text {cr }}$ $\sim\left(G M_{\text {lens }} / c^{3}\right)^{-1} \sim 10^{4}\left(M_{\text {lens }} / M_{\odot}\right)^{-1} \mathrm{~Hz}$. For example, the amplification effect is almost negligible for waves with $f$ $\ll f_{\text {cr }}$ where the geometrical optics approximation is not valid [26-28]. There might be an effective time delay between the gravitational wave and the long wave limit (electromagnetic waves) at most $G M_{\text {lens }} / c^{3} \sim 10^{-4} \mathrm{~s}$ with a phase shift at the level of $\sim 10^{-6}$ rad. This, however, is much smaller than our resolution $\Delta \phi /(2 \pi f) \sim 10 \mathrm{~s}$ and well below the phase measurement error. Given the low probability for lensing and the small phase difference expected, we do not consider lensing contamination to be a major source of concern.

Other potential contaminants include modifications to the speed of light, relative to gravity waves, based on refractive fluctuations through the neutral interstellar medium, such as gas clouds. Gravity waves will pass such clouds unobscured, while density gradients within the medium will lead to refraction and, thus, modifications to the path length of the optical light. Here, again, the expectations are of relatively small modifications, if any, but we suggest that further studies may be necessary given the importance of combined GW and optical studies in the LISA era and the unique sample of CWDBs that LISA data are expected to provide.

\section{SUMMARY}

The arrival times of gravitational waves and optical light from orbiting binaries provide a mechanism for understanding the propagation effects of gravity, when compared to light. This is easily achieved via binary orbital phase measurement and by looking for a phase offset in the optically derived orbital phase related to that derived from gravity wave data. Using a sample of close white dwarf binaries detectable with the Laser Interferometer Space Antenna and the associated optical light curve data related to binary eclipses, we determine the accuracy to which orbital phase difference can be extracted. We consider an application of these measurements, determining an upper limit on the graviton mass.

For a subsample of $\sim 400 \mathrm{CWDBs}$ with high signal-tonoise ratio gravity wave and optical data with magnitudes brighter than 25 , the combined upper limit on the graviton mass is at the level of $\sim 6 \times 10^{-24} \mathrm{eV}$, which is two orders of magnitude better than the limit derived by Yukawacorrection arguments related to the Newtonian potential and applied to the Solar System.

\section{ACKNOWLEDGMENT}

We thank Alison Farmer for information related to the binary sample and Daniel Holz for pointing out previous work on this subject. This work was supported in part by DOE Grant DE-FG03-92-ER40701 and the Sherman Fairchild foundation (AC) and by NASA Grant NAG5-10707 and the Japan Society for the Promotion of Science (NS).
[1] A. Cooray, A. Farmer, and N. Seto, Astrophys. J. Lett. 601, L47 (2004).

[2] R.F. Webbink and Z. Han, in Laser Interferometer Space Antenna, edited by W. M. Folkner, AIP Conf. Proc. No. 456 (AIP, Woodbury, NY, 1998), p. 61.

[3] G. Nelemans, L.R. Yungelson, and S.F. Portegies Zwart, Astron. Astrophys. 375, 890 (2001).

[4] N. Seto, Mon. Not. R. Astron. Soc. 333, 469 (2002).

[5] C.M. Will, Phys. Rev. D 57, 2061 (1998).

[6] C.M. Will, Class. Quantum Grav. 20, S219 (2003).

[7] S.L. Larson and W.A. Hiscock, Phys. Rev. D 61, 104008 (2000).

[8] C. Cutler, W.A. Hiscock, and S.L. Larson, Phys. Rev. D 67, 024015 (2003).

[9] C. Cutler and E. Flanagan, Phys. Rev. D 49, 2658 (1994); C. Cutler, ibid. 57, 7089 (1998).

[10] P.C. Peters, Phys. Rev. D 136, 1224 (1964).

[11] A. Farmer and E.S. Phinney, Mon. Not. R. Astron. Soc. 346, 1197 (2003).

[12] G. Nelemans, L.R. Yungelson, S.F. Portegies Zwart, and F. Verbunt, Astron. Astrophys. 365, 492 (2001).
[13] R. Takahashi and N. Seto, Astrophys. J. 575, 1030 (2002).

[14] N.J. Cornish and S.L. Larson, Phys. Rev. D 67, 103001 (2003).

[15] M. Nauenberg, Astrophys. J. 175, 417 (1972).

[16] P.F. Maxted, T.R. Marsh, and C.K. Moran, Mon. Not. R. Astron. Soc. 332, 745 (2002).

[17] N. Seto, Phys. Rev. D 66, 122001 (2002).

[18] L.S. Finn and K.S. Thorne, Phys. Rev. D 62, 124021 (2000).

[19] J.N. Bahcall and R.M. Soneira, Astrophys. J., Suppl. Ser. 44, 73 (1980).

[20] I. Iben, A.V. Tutukov, and A.V. Fedorova, Astrophys. J. 503, 344 (1998)

[21] C. Talmadge, J.-P. Berthias, R.W. Hellings, and E.M. Standish, Phys. Rev. Lett. 61, 1159 (1988).

[22] A.S. Goldhaber and N.M. Nieto, Phys. Rev. D 9, 1119 (1974).

[23] M.G. Hare, Can. J. Phys. 51, 431 (1973).

[24] P.J. Sutton and L.S. Finn, Class. Quantum Grav. 19, 1355 (2002).

[25] C. Alcock et al., Astrophys. J. 541, 734 (2000).

[26] T.T. Nakamura, Phys. Rev. Lett. 80, 1138 (1998).

[27] R. Takahashi and T. Nakamura, Astrophys. J. 595, 1039 (2003).

[28] K. Yamamoto, astro-ph/0309696. 